JOURNAL OF INTEGRAL EQUATIONS

AND APPLICATIONS

Volume 2, Number 3, Summer 1990

\title{
GLOBAL EXISTENCE IN ONE-DIMENSIONAL NONLINEAR VISCOELASTICITY WITH HEAT CONDUCTION
}

\author{
MORTIN E. GURTIN AND WILLIAM J. HRUSA
}

Dedicated to our good friend John Nohel on his 65th birthday

1. Introduction. In motions of nonlinear viscoelastic materials the dissipative effects due to memory restrain disturbances of small amplitude, but the destabilizing effects of nonlinearity in the instantaneous response are dominant for disturbances of large amplitude. The difficulties inherent in the study of general materials with memory have led to an interest in models that capture the interaction between dissipation and nonlinearity, but are sufficiently simple to allow analysis of corresponding history-value problems. In one space dimension a class of mechanical models with this property is based on single-integral laws,

$$
\sigma(t)=\hat{\sigma}(\varepsilon(t))+\int_{0}^{\infty} \tilde{\sigma}(\varepsilon(t), \varepsilon(t-s), s) d s,
$$

giving the stress $\sigma(t)$ when the strain $\varepsilon(\tau)$ is known at all past times $\tau \leq t$.

There is a large literature (cf., e.g., [13] and the references cited therein) concerning history-value problems associated with (1.1); in particular, under physically natural assumptions on $\hat{\sigma}$ and $\tilde{\sigma}$, a globally defined smooth solution exists provided the data are smooth and sufficiently close to equilibrium. Similar results have been obtained for rigid heat conductors of single-integral type (cf., e.g., [1]) and for thermoelastic heat conductors (cf., e.g., $[\mathbf{8}, \mathbf{1 4}, \mathbf{1 6}]$ ). However, to our knowledge there are no general results applicable to nonlinear materials of single-integral type for deforming bodies under varying temperature. Our objective here is to study such problems using, as a basis, a thermodynamic theory of viscoelastic materials of singleintegral type developed in [6]. (A general theory of thermodynamics of materials with memory was developed earlier by Coleman $[\mathbf{2}]$. Much of Coleman's theory applies to single-integral laws; however, because single-integral laws have such restricted form, the corresponding set of thermodynamical restrictions is richer than the set originally derived by

Copyright (C)1990 Rocky Mountain Mathematics Consortium 
Coleman). In particular, we consider materials with stress and energy related to strain and temperature through constitutive equations of the form (1.1), and with heat flux given by a nonlinear Fourier law. We prove global existence of a classical solution for the history-value problem corresponding to the following conditions: the body for $t \leq 0$ and the boundary for $t>0$ are displacement free and at constant temperature $\theta_{0}$; the body force and heat supply are smooth and small for $t>0$.

The underlying thermodynamic structure is the basic ingredient of our theory: compatibility of the constitutive equations with the second law provides basic inequalities and a symmetry of cross derivatives that are essential to our argument.

\section{Formulation of the history-value problem.}

2.1. Notation. We will consistently use the following notation: $\langle\cdot, \cdot\rangle$ and $|\cdot|$ are the standard inner product and norm on $\mathbf{R}^{2}$; for functions $f\left(a_{1}, a_{2}, a_{3}, \ldots\right)$ of several variables, $f_{, i}=\partial f / \partial a_{i}$; for functions $f(x, t)$ of position $x$ and time $t, f_{x}=\partial f / \partial x, f^{\cdot}=\partial f / \partial t, D^{n} f$ is a formal list of the $n$th order partial derivatives of $f$, and $\left|D^{n} f\right|$ designates the pointwise Euclidean norm of $D^{n} f$. A dot will also denote differentiation with respect to a single time-like variable.

The symbol "*" denotes convolution:

$$
(a * f)(t)=\int_{0}^{t} a(t-\tau) f(\tau) d \tau .
$$

(For functions of position and time " $*$ " will be with respect to time.)

Let $T \in \mathbf{R}$ and $f:(-\infty, T] \rightarrow \mathbf{R}$ be given. Then the history $f^{t}:[0, \infty) \rightarrow \mathbf{R}$ of $f$ up to time $t \in(-\infty, T]$ is defined by

$$
f^{t}(s)=f(t-s), \quad s \geq 0 .
$$

2.2. The formal history-value problem. Consider-under an appropriate scaling - a one-dimensional homogeneous body $\mathcal{B}$ identified with the interval $[0,1]$ it occupies in a homogeneous reference configuration of unit density. The thermomechanics of $\mathcal{B}$ is described by seven func- 
tions of position $x \in[0,1]$ and time $t$,

$$
\begin{aligned}
& u(x, t) \text { displacement, } \\
& \sigma(x, t) \text { stress, } \\
& f(x, t) \text { body force, } \\
& e(x, t) \text { internal energy, } \\
& \theta(x, t) \text { absolute temperature, } \\
& q(x, t) \text { heat flux, } \\
& r(x, t) \quad \text { heat supply, }
\end{aligned}
$$

related through balance laws for momentum and energy:

$$
\begin{aligned}
u^{*} & =\sigma_{x}+f, \\
e & =-q_{x}+\sigma u_{x}+r .
\end{aligned}
$$

We consider constitutive equations in the form of single-integral laws for stress and internal energy in conjunction with a Fourier law for heat conduction. Precisely, writing

$$
\varepsilon=u_{x}, \quad \gamma=\theta_{x}
$$

for the strain and temperature gradient, we suppose that

$$
\begin{aligned}
& \sigma(t)=\hat{\sigma}(\varepsilon(t), \theta(t))+\int_{0}^{\infty} \tilde{\sigma}(\varepsilon(t), \theta(t), \varepsilon(t-s), \theta(t-s), s) d s, \\
& e(t)=\hat{e}(\varepsilon(t), \theta(t))+\int_{0}^{\infty} \tilde{e}(\varepsilon(t), \theta(t), \varepsilon(t-s), \theta(t-s), s) d s, \\
& q(t)=-\kappa(\varepsilon(t), \theta(t)) \gamma(t),
\end{aligned}
$$

with response functions $\hat{\sigma}, \tilde{\sigma}, \hat{e}, \tilde{e}$, and $\kappa$ independent of $x$.

We assume that initially and for all past times the body is displacement free and at constant temperature $\theta_{0}$, and that the same conditions hold on the boundary for all positive time. The history-value problem under consideration then consists in solving (2.1)-(2.3) subject to the boundary conditions

$$
u(0, t)=u(1, t)=0, \quad \theta(0, t)=\theta(1, t)=\theta_{0}, \quad t \geq 0,
$$


and the initial conditions

$$
\begin{aligned}
& u(x, t)=0, \quad \theta(x, t)=\theta_{0}, \quad x \in[0,1], t<0, \\
& u(x, 0)=u(x, 0)=0, \quad \theta(x, 0)=\theta_{0}, \quad x \in[0,1],
\end{aligned}
$$

with $\theta_{0}>0$ a prescribed constant.

2.3. Assumptions concerning the constitutive equations and data. We normalize the integrands in (2.3) so that

$$
\tilde{\sigma}(\alpha, \beta, \alpha, \beta, s)=\tilde{e}(\alpha, \beta, \alpha, \beta, s)=0 ;
$$

the functions $\hat{\sigma}$ and $\hat{e}$ then define the equilibrium response of the material. By (2.6), for $\tilde{f}$ equal to $\tilde{\sigma}$ or $\tilde{e}$,

$$
\begin{aligned}
& \tilde{f}_{, 1}(\alpha, \beta, \alpha, \beta, s)=-\tilde{f}_{, 3}(\alpha, \beta, \alpha, \beta, s), \\
& \tilde{f}_{, 2}(\alpha, \beta, \alpha, \beta, s)=-\tilde{f}_{, 4}(\alpha, \beta, \alpha, \beta, s) .
\end{aligned}
$$

Some notation is useful. We write

$$
U=(-1, \infty) \times(0, \infty)
$$

for the set of strain-temperature pairs, and we refer to a function $\mu: U \times U \times[0, \infty) \rightarrow \mathbf{R}$ as locally dominated if, for every compact set $Z \subset U \times U$, there is a function $k \in L^{1}[0, \infty)$ such that

$$
|\mu(\varepsilon, \theta, \alpha, \beta, s)| \leq k(s) \quad \forall(\varepsilon, \theta, \alpha, \beta) \in Z, s \in[0, \infty) .
$$

In addition, we refer to the pair $\left(0, \theta_{0}\right)$ as the equilibrium state and write

$$
\Delta \mu(\varepsilon, \theta, \alpha, \beta, s)=\mu(\varepsilon, \theta, \alpha, \beta, s)-\mu\left(0, \theta_{0}, 0, \theta_{0}, s\right)
$$

for the departure of $\mu$ from equilibrium.

We assume that the response functions are consistent with the following smoothness hypotheses:

$$
\begin{aligned}
& \hat{\sigma}, \hat{\varepsilon}, \kappa \in C^{3}(U) ; \\
& \tilde{\sigma}, \tilde{\varepsilon} \in C^{3}(U \times U \times[0, \infty)) ; \tilde{\sigma}, \tilde{\varepsilon} \text { and their partial } \\
& \text { derivatives through order } 3 \text { are locally dominated. }
\end{aligned}
$$


This assumption can be relaxed, but general assumptions under which our existence proof applies are complicated to state; we believe that (A1) is reasonable from the viewpoint of mechanics. (It would be of interest to develop an existence theory for kernels $\tilde{\sigma}(\varepsilon, \theta, \alpha, \beta, s)$ and $\tilde{e}(\varepsilon, \theta, \alpha, \beta, s)$ that are singular at $s=0$; to treat such kernels within our framework would require major modifications.)

The moduli

$$
\begin{aligned}
A_{\mathrm{eq}}(\varepsilon, \theta) & =\hat{\sigma}_{, 1}(\varepsilon, \theta), & B_{\mathrm{eq}}(\varepsilon, \theta) & =\hat{\sigma}_{, 2}(\varepsilon, \theta), \\
M_{\mathrm{eq}}(\varepsilon, \theta) & =\hat{e}_{, 1}(\varepsilon, \theta), \quad & C_{\mathrm{eq}}(\varepsilon, \theta) & =\hat{e}_{, 2}(\varepsilon, \theta),
\end{aligned}
$$

characterize the equilibrium response of the material at a straintemperature pair $(\varepsilon, \theta) \in U$, while the functionals

$$
\begin{aligned}
& A_{\text {inst }}\left(\varepsilon^{t}, \theta^{t}\right)=A_{\text {eq }}(\varepsilon(t), \theta(t))+\int_{0}^{\infty} \tilde{\sigma}_{, 1}(\varepsilon(t), \theta(t), \varepsilon(t-s), \theta(t-s), s) d s, \\
& B_{\text {inst }}\left(\varepsilon^{t}, \theta^{t}\right)=B_{\text {eq }}(\varepsilon(t), \theta(t))+\int_{0}^{\infty} \tilde{\sigma}_{, 2}(\varepsilon(t), \theta(t), \varepsilon(t-s), \theta(t-s), s) d s, \\
& M_{\text {inst }}\left(\varepsilon^{t}, \theta^{t}\right)=M_{\text {eq }}(\varepsilon(t), \theta(t))+\int_{0}^{\infty} \tilde{e}_{, 1}(\varepsilon(t), \theta(t), \varepsilon(t-s), \theta(t-s), s) d s, \\
& C_{\text {inst }}\left(\varepsilon^{t}, \theta^{t}\right)=C_{\text {eq }}(\varepsilon(t), \theta(t))+\int_{0}^{\infty} \tilde{e}_{, 2}(\varepsilon(t), \theta(t), \varepsilon(t-s), \theta(t-s), s) d s .
\end{aligned}
$$

characterize the instantaneous response at the history-pair $\left(\varepsilon^{t}, \theta^{t}\right)$. We will refer to these functionals as instantaneous derivatives; $A_{\text {inst }}\left(\varepsilon^{t}, \theta^{t}\right)$ represents the derivative of the stress at time $t$ with respect to the present strain $\varepsilon(t)$ holding the past strain values $\varepsilon(\tau), \tau<t$ and the entire history $\theta^{t}$ fixed, and so forth.

To write the functional differential equations that define our theory we need expressions for the functional derivatives of the constitutive relations for the stress and energy. These derivatives are linear functions of the strain and temperature "perturbations" $f$ and $g$; for $\mathrm{f}, \mathrm{g}:(-\infty, T] \rightarrow \mathbf{R}$ continuous functions with

$$
\mathrm{f}=\mathrm{g}=0 \quad \text { on }(-\infty, 0],
$$

the functional derivative of the stress is given by 


$$
\begin{aligned}
& A_{\text {inst }}\left(\varepsilon^{t}, \theta^{t}\right) \mathrm{f}(t)+B_{\text {inst }}\left(\varepsilon^{t}, \theta^{t}\right) \mathrm{g}(t) \\
&+\int_{0}^{t} \tilde{\sigma}_{, 3}(\varepsilon(t), \theta(t), \varepsilon(t-s), \theta(t-s), s) \mathrm{f}(t-s) d s \\
&+\int_{0}^{t} \tilde{\sigma}_{, 4}(\varepsilon(t), \theta(t), \varepsilon(t-s), \theta(t-s), s) \mathrm{g}(t-s) d s, \\
& t>0,
\end{aligned}
$$

and a similar expression holds for the energy.

The functions $a, b, m, c:[0, \infty) \rightarrow \mathbf{R}$ defined by

$$
\begin{aligned}
a^{\prime}(s) & =\tilde{\sigma}_{, 3}\left(0, \theta_{0}, 0, \theta_{0}, s\right), & a(\infty) & =0, \\
b^{\prime}(s) & =\tilde{\sigma}_{, 4}\left(0, \theta_{0}, 0, \theta_{0}, s\right), & b(\infty) & =0 \\
m^{\prime}(s) & =\tilde{e}_{, 3}\left(0, \theta_{0}, 0, \theta_{0}, s\right), & m(\infty) & =0 \\
c^{\prime}(s) & =\tilde{e}_{, 4}\left(0, \theta_{0}, 0, \theta_{0}, s\right), & c(\infty) & =0
\end{aligned}
$$

characterize the hereditary behavior of the material near the equilibrium state $\left(0, \theta_{0}\right)$. (It is more customary to use the stress-strain relaxation function $G(s), 0 \leq s<\infty$, defined by $G^{\prime}(s)=a^{\prime}(s), G(\infty)=$ $A_{\text {eq }}\left(0, \theta_{0}\right)$; in fact, the results of [6] are phrased in terms of the relaxation (matrix-) function $\mathcal{G}(s)$ with $\mathcal{G}^{\prime}(s)=\mathcal{A}^{\prime}(s)$ (cf. (2.23)) and $\mathcal{G}(\infty)$ defined in terms of the equilibrium moduli $(2.11)$ at $\left(0, \theta_{0}\right)$.) By $(2.7)$,

$$
p(0)=P_{\text {inst }}\left(0, \theta_{0}\right)-P_{\text {eq }}\left(0, \theta_{0}\right),
$$

where here and in what follows,

$$
\begin{gathered}
P \text { stands for any one of the letters } A, B, M, C \text { and } \\
p \text { for the corresponsing lower case letter } a, b, m \text {, or } c,
\end{gathered}
$$

and where $P_{\text {inst }}\left(0, \theta_{0}\right)$ means $P_{\text {inst }}$ evaluated at the constant historypair with value $\left(0, \theta_{0}\right)$.

We assume that

$$
a, b, m, c \in L^{1}[0, \infty) .
$$


The assumptions (A1) and (A2) imply that

$$
P_{\text {eq }} \in C^{2}(U), \quad p \in C^{3}[0, \infty) \cap W^{3,1}[0, \infty) .
$$

We assume, in addition, that - at the equilibrium state $\left(0, \theta_{0}\right)$ - the equilibrium elasticity, the instantaneous specific heat, and the conductivity are strictly positive:

$$
A_{\text {eq }}\left(0, \theta_{0}\right)>0, \quad C_{\text {inst }}\left(0, \theta_{0}\right)>0, \quad \kappa\left(0, \theta_{0}\right)>0 .
$$

In analyzing the behavior of the material near the equilibrium state $\left(0, \theta_{0}\right)$, it is convenient to write the functional derivatives for stress and energy using - in place of the instantaneous derivatives - the functionals

$$
\begin{aligned}
P\left(\varepsilon^{t}, \theta^{t}\right) & =P_{\text {inst }}\left(\varepsilon^{t}, \theta^{t}\right)-p(0) \\
& =P_{\text {eq }}\left(0, \theta_{0}\right)+P_{\text {inst }}\left(\varepsilon^{t}, \theta^{t}\right)-P_{\text {inst }}\left(0, \theta_{0}\right) .
\end{aligned}
$$

Using these definitions, we can express the functional derivative (2.13) in the form

$$
\begin{aligned}
A\left(\varepsilon^{t}, \theta^{t}\right) \mathrm{f}(t) & +B\left(\varepsilon^{t}, \theta^{t}\right) \mathrm{g}(t) \\
& +(a * \mathrm{f})^{\cdot}(t)+(b * \mathrm{~g})^{\cdot}(t)+J \tilde{\sigma}\left(\varepsilon^{t}, \theta^{t} ; \mathbf{f}^{t}, \mathbf{g}^{t}\right),
\end{aligned}
$$

where

$$
\begin{aligned}
J \tilde{\sigma}\left(\varepsilon^{t}, \theta^{t} ; \mathrm{f}^{t}, \mathrm{~g}^{t}\right) \\
=\int_{0}^{t} \Delta\left(\tilde{\sigma}_{, 3}\right)(\varepsilon(t), \theta(t), \varepsilon(t-s), \theta(t-s), s) \mathrm{f}(t-s) d s \\
\quad+\int_{0}^{t} \Delta\left(\tilde{\sigma}_{, 4}\right)(\varepsilon(t), \theta(t), \varepsilon(t-s), \theta(t-s), s) \mathrm{g}(t-s) d s .
\end{aligned}
$$

Similarly, the functional derivative of the energy is given by

$$
\begin{aligned}
M\left(\varepsilon^{t}, \theta^{t}\right) \mathrm{f}(t) & +C\left(\varepsilon^{t}, \theta^{t}\right) \mathrm{g}(t) \\
& +(m * \mathrm{f})^{\cdot}(t)+(c * \mathrm{~g})^{\cdot}(t)+J \tilde{e}\left(\varepsilon^{t}, \theta^{t} ; \mathrm{f}^{t}, \mathrm{~g}^{t}\right),
\end{aligned}
$$

with $J \tilde{e}\left(\varepsilon^{t}, \theta^{t} ; \mathrm{f}^{t}, \mathrm{~g}^{t}\right)$ defined by a relation strictly analogous to (2.19). 
Thermodynamics places restrictions on the constitutive equations, and these will be important in our analysis. In particular, we have shown [6] that derivatives with respect to temperature of the stress response functions and derivatives with respect to strain of the energy response functions satisfy

$$
\begin{aligned}
\hat{e}_{, 1}(\varepsilon, \theta) & =\hat{\sigma}(\varepsilon, \theta)-\theta \hat{\sigma}_{, 2}(\varepsilon, \theta), \\
\tilde{e}_{, 1}(\varepsilon, \theta, \alpha, \beta, s) & =\tilde{\sigma}(\varepsilon, \theta, \alpha, \beta, s)-\theta \tilde{\sigma}_{, 2}(\varepsilon, \theta, \alpha, \beta, s),
\end{aligned}
$$

which we here take as hypotheses. By (2.3), (2.7), (2.11), and (2.14), (A4) yields

$$
\begin{aligned}
M_{\mathrm{eq}}(\varepsilon, \theta) & =\hat{\sigma}(\varepsilon, \theta)-\theta B_{\mathrm{eq}}(\varepsilon, \theta), \quad m=-\theta_{0} b, \\
M_{\text {inst }}\left(\varepsilon^{t}, \theta^{t}\right) & =\sigma(t)-\theta(t) B_{\text {inst }}\left(\varepsilon^{t}, \theta^{t}\right) .
\end{aligned}
$$

In addition, letting

$$
g(s)=-\theta_{0}^{-1} c(s),
$$

we have shown that the matrix function

$$
\mathcal{A}(s)=\left[\begin{array}{ll}
a(s) & b(s) \\
b(s) & g(s)
\end{array}\right], \quad s \geq 0,
$$

has first derivative negative semi-definite and second derivative positive semi-definite for all $s \geq 0$. Here, we assume that

$$
\begin{aligned}
& \mathcal{A}^{\prime}(0) \text { is negative definite, } \\
& \mathcal{A}^{\prime \prime}(s) \text { is positive semi-definite for all } s \geq 0 .
\end{aligned}
$$

The hereditary nature of the constitutive equations for stress and energy gives rise to dissipation. A measure of this dissipationappropriate to small departures from the equilibrium state $\left(0, \theta_{0}\right)$-is given by the quadratic form

$$
Q(\Phi)(t)=\int_{0}^{t}\langle\Phi(s),(\mathcal{A} * \Phi)(s)\rangle d s
$$


defined for all $t \geq 0$ and all locally square-integrable function $\Phi$ : $[0, \infty) \rightarrow \mathbf{R}^{2}$, where

$$
(\mathcal{A} * \Phi)(s)=\int_{0}^{s} \mathcal{A}(s-\tau) \Phi(\tau) d \tau .
$$

Here $\Phi$ should be interpreted as a perturbation $\Phi=\left(\mathrm{f}^{\prime}, \mathrm{g}^{*}\right)$ in strainrate and temperature-rate. As is known, (A5) yields the condition $Q(\Phi)(t) \geq 0$ (cf., e.g., $[\mathbf{4}, \mathbf{5}])$.

Finally, the data $f$ and $r$ are assumed to satisfy the following compatibility and smoothness conditions:

$$
\begin{aligned}
& f, D^{1} f, f_{x}^{\cdot}, f^{*}, r, D^{1} r, r_{x}^{\cdot}, r^{*} \in L^{2}\left([0, \infty) ; L^{2}(0,1)\right), \\
& r(\cdot, 0) \in H^{2}(0,1), \\
& f(0,0)=f(1,0)=0, \quad r(0,0)=r(1,0)=0 .
\end{aligned}
$$

In (A6) and in the theorem stated below, derivatives should be interpreted in the sense of distributions.

2.4. The existence theorem and the main ingredients of its proof. Our main result is the following theorem, in which we measure the size of the data using the function

$$
\begin{aligned}
\mathcal{J}(f, r)= & \int_{0}^{\infty} \int_{0}^{1}\left\{f^{2}+\left|D^{1} f\right|^{2}+f_{x}^{\cdot 2}+f^{. \cdot 2}+r^{2}+\left|D^{1} r\right|^{2}+r_{x}^{\cdot 2}+r^{. \cdot 2}\right\} \\
& (x, t) d x d t \\
& +\int_{0}^{1} r_{x x}(x, 0)^{2} d x .
\end{aligned}
$$

Existence Theorem. Consider the history-value problem defined by (2.1)-(2.5) with constitutive functions subject to assumptions (A1)(A5). There is a constant $\mu>0$ such that, for all $f, r$ consistent with (A6) and

$$
\mathcal{J}(f, r) \leq \mu
$$

this problem has a unique solution $(u, \theta)$ with the following properties: 
(i) $\left(u_{x}(x, t), \theta(x, t)\right) \in U$ for all $x \in[0,1], t \geq 0$;

(ii) $u, D^{1} u, D^{2} u, D^{3} u, \theta-\theta_{0}, D^{1} \theta, D^{2} \theta, \theta_{x x x}$ and $\theta_{x x}$ belong to

$C\left([0, \infty) ; L^{2}(0,1)\right)$, to $L^{\infty}\left([0, \infty) ; L^{2}(0,1)\right)$, and to $L^{2}\left([0, \infty) ; L^{2}(0,1)\right)$;

$\theta_{\ddot{x}}^{*}$ belongs to $L^{2}\left([0, \infty) ; L^{2}(0,1)\right)$.

Moreover, as $t \rightarrow \infty$,

$$
u, D^{1} u, D^{2} u, \theta-\theta_{0}, D^{1} \theta, \theta_{x x}, \theta_{x} \rightarrow 0 \text { uniformly on }[0,1] .
$$

REMARK. Our proof of the theorem is valid under slightly weaker assumptions on $f$ and $r$ (e.g., nothing need be assumed concerning $f_{x}^{\prime}$ and $r_{x}^{\cdot}$ provided we impose stronger restrictions on $f_{x}$ and $r_{x}$ ). Moreover, solutions with higher regularity can be obtained by assuming greater regularity for the constitutive functions and the data and more stringent compatibility for the data at the boundary. Such modifications are similar to the isothermal case (cf., e.g., $[\mathbf{3}]$ ).

REMARK. The arguments we use to prove the existence theorem can also be used to treat the following: the boundary conditions $u=0, q=$ 0 ; the boundary conditions $\sigma=0, \theta=\theta_{0}$; initial data of the form

$$
\begin{aligned}
& u(x, t)=U(x, t), \quad \theta(x, t)=\Theta(x, t), \quad x \in[0,1], \quad t<0, \\
& u(x, 0)=U_{0}(x), \quad u(x, 0)=U_{1}(x), \quad \theta(x, 0)=\Theta_{0}(x), \quad x \in[0,1],
\end{aligned}
$$

under suitable assumptions of smoothness, smallness, and compatibility with the boundary conditions.

Our proof involves the functional-differential equation that results when the constitutive relations (2.3) are combined with the balance laws (2.1). The term $\sigma_{x}$ in $(2.1)_{1}$ is given by (2.18) with $\mathrm{f}=u_{x x}, \mathrm{~g}=$ $\theta_{x}$, while $e$ in $(2.1)_{2}$ is given by $(2.20)$ with $\mathrm{f}=u_{x}, \mathrm{~g}=\theta$; thus, in view of (2.21) and (2.22), (2.1) and (2.3) combine to give the following 
equations, in which the argument $(x, t)$ has, for convenience, been omitted:

$$
\begin{aligned}
u^{* .}=A\left(u_{x}^{t}, \theta^{t}\right) u_{x x} & +B\left(u_{x}^{t}, \theta^{t}\right) \theta_{x}+\left(a * u_{x x}\right)^{\cdot}+\left(b * \theta_{x}\right)^{\cdot} \\
& +f+J \tilde{\sigma}\left(u_{x}^{t}, \theta^{t} ; u_{x x}^{t}, \theta_{x}^{t}\right), \\
G\left(u_{x}^{t}, \theta^{t}\right) \theta^{\cdot}= & \left(K\left(u_{x}, \theta\right) \theta_{x}\right) x_{x}+B\left(u_{x}^{t}, \theta^{t}\right) u_{x}^{\cdot}+\left(b * u_{x}^{\cdot}\right)^{\cdot}+\left(g * \theta^{\cdot}\right) \\
& +S\left(u_{x}^{t}, \theta^{t}\right) u_{x}+\theta_{0}^{-1}\left[r-J \tilde{e}\left(u_{x}^{t}, \theta^{t} ;\left(u_{x}\right)^{t},\left(\theta^{\cdot}\right)^{t}\right)\right],
\end{aligned}
$$

where

$$
\begin{aligned}
& K=\theta_{0}^{-1} \kappa, \quad G=\theta_{0}^{-1} C, \\
& S\left(u_{x}^{t}, \theta^{t}\right)=\theta_{0}^{-1}\left(\theta-\theta_{0}\right) B_{\text {inst }}\left(u_{x}^{t}, \theta^{t}\right) .
\end{aligned}
$$

To prove the existence theorem we derive a priori estimates that can be used to continue a local solution globally in time. For this purpose it is convenient to consider the system

$$
\begin{aligned}
& w^{*}=A\left(u_{x}^{t}, \theta^{t}\right) w_{x x}+B\left(u_{x}^{t}, \theta^{t}\right) \varphi_{x}+\left(a * w_{x x}\right)^{\cdot}+\left(b * \varphi_{x}\right)^{*}+F \\
& \begin{array}{r}
G\left(u_{x}^{t}, \theta^{t}\right) \varphi^{\cdot}=\left[K\left(u_{x}, \theta\right) \varphi_{x}\right]_{x}+B\left(u_{x}^{t}, \theta^{t}\right) w_{x}^{\cdot}+\left(b * w_{x}^{\cdot}\right)^{*}+\left(g * \varphi^{\cdot}\right)^{*} \\
+S\left(u_{x}^{t}, \theta^{t}\right) w_{x}^{\cdot}+R
\end{array}
\end{aligned}
$$

with

$$
\begin{aligned}
& F=f+J \tilde{\sigma}\left(u_{x}^{t}, \theta^{t} ; u_{x x}^{t}, \theta_{x}^{t}\right), \\
& R=\theta_{0}^{-1}\left[r-J \tilde{e}\left(u_{x}^{t}, \theta^{t} ;\left(u_{x}^{*}\right)^{t},\left(\theta^{*}\right)^{t}\right)\right],
\end{aligned}
$$

together with homogeneous Dirichlet boundary conditions and zero initial data for $w$ and $\varphi$. Note that (2.32) is linear in $w$ and $\varphi$. We shall obtain estimates for $w$ and $\varphi$ in terms of $u, \theta, f$, and $r$, and then put $w=u$ and $\varphi=\theta-\theta_{0}$. Here is it important to note that, by (2.19) and its counterpart for energy, $F$ and $R$ depend linearly on $u_{x x}^{t}(s), \theta_{x}^{t}(s),\left(u_{x}^{\cdot}\right)^{t}(s)$, and $\left(\theta^{\cdot}\right)^{t}(s)$ through integrals with respect to $s$ against kernels that are smooth functions of $u_{x}(t), \theta(t), u_{x}(t-s), \theta(t-s)$, and $s$. Thus $F$ and $R$ will have higher temporal regularity than $u_{x x}, \theta_{x}, u_{x}$, and $\theta$. When we derive a priori estimates, this fact allows us to treat the terms associated with $F$ and $R$ in a very simple manner. 
Note that, by (2.17), (2.22), and $(2.31)_{1,2}$, the conditions (A3) have the form

$$
A\left(0, \theta_{0}\right)>0, \quad G\left(0, \theta_{0}\right)>g(0), \quad \kappa\left(0, \theta_{0}\right)>0 .
$$

There are three main ingredients in the proof of the existence theorem. The first - a consequence of the equations (2.32) and the initial/boundary conditions for $w$ and $\varphi$-is the energy identity

$$
\mathcal{U}(w, \varphi)+\mathcal{V}(w, \varphi)=\mathcal{F}(F, R, w, \varphi)+\mathcal{N}_{0}(w, \varphi),
$$

with

$$
\begin{array}{r}
\mathcal{U}(w, \varphi)(t)=\frac{1}{2} \int_{0}^{1}\left(w^{\cdot 2}+A w_{x}^{2}\right)(x, t) d x+\int_{0}^{t} \int_{0}^{1}\left(K \varphi_{x}^{2}\right)(x, s) d x d s \\
\mathcal{V}(w, \varphi)(t)=\int_{0}^{1}\left\{\frac{1}{2} G \varphi^{2}+Q\left(w_{x}^{\cdot}, \varphi^{\cdot}\right)-\varphi\left[b * w_{x}^{\cdot}+g * \varphi^{\cdot}\right]\right\}(x, t) d x \\
\mathcal{F}(F, R, w, \varphi)(t)=\int_{0}^{t} \int_{0}^{1}\left(F w^{\cdot}+R \varphi\right)(x, s) d x d s \\
\mathcal{N}_{0}(w, \varphi)(t)=\int_{0}^{t} \int_{0}^{1}\left\{\frac{1}{2} A \cdot w_{x}^{2}-A_{x} w_{x} w^{\cdot}-B_{x} \varphi w^{\cdot}-S_{x} \varphi w^{\cdot}-S \varphi_{x} w^{\cdot}\right. \\
\left.+\frac{1}{2} G \cdot \varphi^{2}\right\}(x, s) d x d s
\end{array}
$$

This identity and analogous identities obtained by time-differentiating (2.32) form our basis for obtaining a priori estimates.

The second ingredient is the inequality

$$
\frac{1}{2} G \varphi^{2}+Q\left(w_{x}^{\cdot}, \varphi^{\cdot}\right)-\varphi\left[b * w_{x}^{\cdot}+g * \varphi^{\cdot}\right] \geq \Lambda\left[\varphi^{2}+Q\left(w_{x}^{\cdot}, \varphi^{\cdot}\right)\right]
$$

( $\Lambda$ a strictly positive constant). This inequality, which is based on ideas of Staffans [15], provides a positive-definite lower bound for $\mathcal{V}(w, \varphi)(t)$. The third ingredient is a related inequality

$$
\int_{0}^{t}|\Phi(s)|^{2} d x \leq \Gamma\left[|\Phi(0)|^{2}+Q(\Phi)(t)+Q\left(\Phi^{\cdot}\right)(t)\right]
$$


based on ideas of Dafermos and Nohel [3] and Staffans [15], which yields bounds for integrals over space and time of squares of various derivatives of $w$ and $\varphi$.

The remainder of the paper is devoted to a proof of the existence theorem. In the next section we give precise statements of the inequalities (2.37) and (2.38). In Section 4 we derive the energy identities discussed above and use them to derive a basic a priori estimate for solutions of the linearized problem. This estimate forms the crux of the proof; to focus on the main ideas behind its derivation, we first restrict attention to the special case in which the coefficients $A, B, G$ and $K$ are constant and $S=0$. In Section 5 we give necessary modifications for nonconstant coefficients. Finally, in Section 6, we complete the proof of the theorem by using the a priori estimates to continue a local solution globally in time. Some of the steps of the proof, particularly those in Sections 5 and 6, are virtually identical to those for the isothermal problem; for such steps the details will generally be omitted (cf., e.g., $[3,13])$.

3. Coercivity of the hereditary dissipation-function $Q(\Phi)$. Let $a, b, g:[0, \infty) \rightarrow \mathbf{R}$ be given. We assume throughout this section that

$$
a, b, g \in C^{3}[0, \infty) \cap W^{3,1}[0, \infty),
$$

and that the matrix function $\mathcal{A}$ defined by (2.23) is consistent with (A5). It then follows from Proposition 16.4.3 of [4] that the matrixvalued kernel $\mathcal{A}$ and the scalar-valued kernels $a$ and $g$ are strongly positive in the sense of Chapter 16 of [4].

For every $T>0$ and every $\Phi \in L^{2}\left([0, T] ; \mathbf{R}^{2}\right)$, we define $Q(\Phi)(t), 0 \leq$ $t \leq T$, through (2.24), (2.25). The fact that $\mathcal{A}$ is strongly positive implies estimates of coercive type for $Q(\Phi)$. Since our energy estimates involve spatial integrals, it is convenient to define the related quadratic form

$\mathcal{Q}(W)(t)=\int_{0}^{t} \int_{0}^{1}\langle W(x, s),(\mathcal{A} * W)(x, s)\rangle d x d s=\int_{0}^{1} Q(W)(x, t) d x$,

for $W \in L^{2}\left([0, T] ; L^{2}\left((0,1) ; \mathbf{R}^{2}\right)\right)$. (The order of integration may be changed by virtue of Fubini's Theorem.) 
The following result is based on ideas of Dafermos and Nohel $[\mathbf{3}]$ and Staffans [15]; the basic idea is that a time-independent bound for

$$
\int_{0}^{t} \int_{0}^{1}|W(x, s)|^{2} d x
$$

follows from time-independent bounds for $\mathcal{Q}(W)$ and $\mathcal{Q}\left(W^{\cdot}\right)$. For technical reasons we use difference quotients

$$
\left(\delta_{h} W\right)(x, t)=h^{-1}[W(x, t+h)-W(x, t)]
$$

in place of derivatives.

LEMMA 3A. There is a constant $\Gamma>0$ such that, for every $T>0$ and every $W \in C\left([0, T] ; L^{2}\left((0,1) ; \mathbf{R}^{2}\right)\right)$,

$$
\begin{aligned}
& \int_{0}^{t} \int_{0}^{1}|W(x, s)|^{2} d x d s \\
& \leq \Gamma\left[\int_{0}^{1}|W(x, 0)|^{2} d x+\mathcal{Q}(W)(t)+\liminf _{h \downarrow 0} \mathcal{Q}\left(\delta_{h} W\right)(t)\right] \\
& \forall t \in[0, T] .
\end{aligned}
$$

An analog of Lemma 3A for scalar-valued kernels is proved in [7]. Lemma 3A follows from Lemma 2.5 of [7] and the following facts: $e^{-t}$ is strongly positive; $\mathcal{A}(t)-\eta I e^{-t}$ is a kernel of positive type for sufficiently small $\eta>0$. (In [7] the spatial interval is $\mathbf{R}$; the same proof applies when the spatial interval is $(0,1)$.)

Our next lemma follows upon taking $y=\Psi(t)$ in the proof of Corollary 16.6 .6 of $[4]$.

Lemma 3B. Choose $T>0$ and $\Phi, \Psi \in L^{2}\left([0, T] ; \mathbf{R}^{2}\right)$. Then

$$
(\langle\Psi,(\mathcal{A} * \Phi)\rangle)^{2} \leq 2\langle\Psi, \mathcal{A}(0) \Psi\rangle Q(\Phi) \quad \text { a.e. on }[0, T] .
$$

Taking $\Psi=(0, z)$ and $\Phi=(v, w)$ in Lemma 3B yields 
Lemma 3C. Choose $T>0$ and $v, w, z \in L^{2}[0, T]$. Then

$$
\{z[b * v+g * w]\}^{2} \leq 2 g(0) z^{2} Q(v, w) \quad \text { a.e. on }[0, T] .
$$

The next result follows from Lemma 3C.

LEMma 3D. Choose $\nu \in \mathbf{R}$ with

$$
\nu>g(0)
$$

Then there is a constant $\Lambda>0$ such that, for every $T>0$ and all $v, w, z \in L^{2}[0, T]$,

$$
\begin{aligned}
& \frac{1}{2} \nu z^{2}+Q(v, w)-z[b * v+g * w] \\
& \geq \Lambda\left[z^{2}+Q(v, w)\right] \quad \text { a.e. on }[0, T] .
\end{aligned}
$$

Lemmas 3A and 3D will be used to obtain a priori bounds from energy integrals. In our analysis energy integrals of higher order will be obtained by differentiating the equations of motion with respect to time, and an additional argument will be needed to obtain bounds for spatial derivatives in terms of quantities previously estimated. Here we will make use of the following lemma (cf. Lemma 3.2 of Dafermos and Nohel] [3] for (i)).

LEMMA 3E. Let $\rho$ be given with

$$
\rho>a(0) .
$$

Let $r:[0, \infty) \rightarrow \mathbf{R}$ denote the unique solution of

$$
\rho r+a^{\prime} * r=-a^{\prime} .
$$

Then

(i) $r \in L^{1}[0, \infty)$;

(ii) for each $z \in L_{\text {loc }}^{1}[0, \infty)$ the function

$$
w=\rho^{-1}(z+r * z)
$$


is the unique solution in $L_{\text {loc }}^{1}[0, \infty)$ of the equation

$$
\rho w+a^{\prime} * w=z ;
$$

(iii) if $z \in L^{p}[0, \infty)$ for some $p \in[1, \infty]$, then $w \in L^{p}[0, \infty)$ and

$$
\|w\|_{p} \leq|\rho|^{-1}\left(1+\|r\|_{1}\right)|| z \|_{p} .
$$

4. The linear system with constant coefficients. The basic energy equations and estimates.

4.1. The linear system. The crucial ideas and estimates behind our proof of existence are more easily discussed in terms of the linear equations (2.32) with $S=0$ and coefficients $A, B, G$, and $K$ constant:

$$
\begin{aligned}
w^{*} & =A w_{x x}+B \varphi_{x}+\left(a * w_{x x}\right)^{\cdot}+\left(b * \varphi_{x}\right)^{\cdot}+F, \\
G \varphi^{\cdot} & =K \varphi_{x x}+B w_{x}^{\cdot}+\left(b * w_{x}^{\cdot}\right)^{\cdot}+\left(g * \varphi^{\cdot}\right)^{\cdot}+R .
\end{aligned}
$$

Here we restrict attention to a fixed time interval $[0, T]$, consider $F$ and $R$ as functions

$$
F(x, t), \quad R(x, t)
$$

of $(x, t) \in[0,1] \times[0, T]$ subject to the smoothness and compatibility requirements

$$
\begin{aligned}
& F, D^{1} F, R, D^{1} R \in C\left([0, T] ; L^{2}(0,1)\right), \\
& R(\cdot, 0) \in H^{2}(0,1), \\
& F^{\cdot *}, R^{*} \in L^{2}\left([0, T] ; L^{2}(0,1)\right), \\
& F(0,0)=F(1,0)=R(0,0)=R(1,0)=0,
\end{aligned}
$$

consider $\varphi$ as the difference

$$
\varphi=\theta-\theta_{0}
$$

and consider boundary and initial conditions of the form

$$
\begin{aligned}
& w(0, t)=w(1, t)=\varphi(0, t)=\varphi(1, t)=0, \quad t \in[0, T], \\
& w(x, 0)=w^{\prime}(x, 0)=\varphi(x, 0)=0, \quad x \in[0,1] .
\end{aligned}
$$


We assume that

$$
\begin{gathered}
A>0, \quad G>g(0), \quad K>0, \\
a, b, g \in C^{3}[0, \infty) \cap W^{3,1}[0, \infty), \quad \mathcal{A} \text { satisfies (A5), }
\end{gathered}
$$

which are all consequences of previous hypotheses (cf. $(2.16)_{2},(\mathrm{~A} 4)$, (2.23), (2.34)). Under these assumptions the problem (4.1), (4.4) has a unique solution $w, \varphi$ satisfying

$$
\begin{gathered}
w, D^{1} w, D^{2} w, D^{3} w, \varphi, D^{1} \varphi, D^{2} \varphi, \varphi_{x x x}, \varphi_{x x}^{\cdot} \in C\left([0, T] ; L^{2}(0,1)\right), \\
\varphi_{x}^{. *} \in L^{2}\left([0, T] ; L^{2}(0,1)\right) .
\end{gathered}
$$

(See Navarro $[\mathbf{1 1}, \mathbf{1 2}]$ for a complete discussion of existence and asymptotic stability for linear thermoviscoelasticity in three dimensions. We need not address the issue of existence for the linear problem: the local existence theorem for the nonlinear problem will ensure that the appropriate linear problem has a solution with the desired regularity.)

4.2. Energy equations. We now derive the energy equations that underlie our analysis. The derivations use the identities

$$
\begin{gathered}
(k * \mathrm{f})^{\cdot}(t)=\left(k * \mathbf{f}^{\cdot}\right)(t)+k(t) \mathbf{f}(0) \\
\int_{0}^{t}(k * \mathrm{f})^{\cdot}(s) \mathrm{g}(s) d s=(k * \mathrm{f})(t) \mathrm{g}(t)-\int_{0}^{t}(k * \mathrm{f})(s) \mathrm{g}^{\cdot}(s) d s
\end{gathered}
$$

We multiply $(4.1)_{1}$ by $w,(4.1)_{2}$ by $\varphi$, add the resulting equations, and integrate with respect to space and time. After a number of integrations by parts using the initial and boundary conditions (4.4), in conjunction with the following intermediate steps based on (4.6),

$$
\begin{aligned}
& \int_{0}^{t} \int_{0}^{1}\left\{\left(a * w_{x x}\right)^{\cdot} w^{\cdot}+\left(b * \varphi_{x}\right)^{\cdot} w^{\cdot}+\left(b * w_{x}^{\cdot}\right)^{\cdot} \varphi+\left(g * \varphi^{\cdot}\right)^{\cdot} \varphi\right\}(x, s) d x d s \\
& =\int_{0}^{t} \int_{0}^{1}\left\{-\left(a * w_{x}^{\cdot}\right) w_{x}^{\cdot}-\left(b * \varphi^{\cdot}\right) w_{x}^{\cdot}-\left(b * w_{x}^{\cdot}\right) \varphi^{\cdot}-\left(g * \varphi^{\cdot}\right) \varphi^{\cdot}\right\}(x, s) d x d s \\
& \quad+\int_{0}^{1}\left\{\left(b * w_{x}^{\cdot}\right) \varphi+\left(g * \varphi^{\cdot}\right) \varphi\right\}(x, t) d x \\
& =\int_{0}^{1}\left\{-Q\left(w_{x}^{\cdot}, \varphi^{\cdot}\right)+\left(b * w_{x}^{\cdot}\right) \varphi+\left(g * \varphi^{\cdot}\right) \varphi\right\}(x, t) d x,
\end{aligned}
$$


we arrive at the desired result: the basic energy equation,

$$
\mathcal{U}(w, \varphi)+\mathcal{V}(w, \varphi)=\mathcal{F}(F, R, w, \varphi)
$$

where $\mathcal{U}, \mathcal{V}$, and $\mathcal{F}$ are as defined in (2.36).

Energy equations involving higher-order derivatives are obtained similarly. We differentiate (4.1) with respect to time, i.e.,

$$
\begin{aligned}
w^{\cdots} & =A w_{x x}^{\cdot}+B \varphi_{x}^{\cdot}+\left(a * w_{x x}\right)^{*}+\left(b * \varphi_{x}\right)^{*}+F^{\cdot}, \\
G \varphi^{*} & =K \varphi_{x x}^{\cdot}+B w_{x}^{\cdot *}+\left(b * w_{x}^{\cdot}\right)^{*}+\left(g * \varphi^{\cdot}\right)^{*}+R
\end{aligned}
$$

multiply $(4.9)_{1}$ by $w^{\prime \prime},(4.9)_{2}$ by $\varphi^{\prime}$, add the resulting equations, and integrate with respect to space and time. Since

$$
\begin{aligned}
& \int_{0}^{t} \int_{0}^{1}\left\{\left(a * w_{x x}\right)^{\cdot *} w^{\cdot \cdot}+\left(b * \varphi_{x}\right)^{\cdot *} w^{\cdot \cdot}+\left(b * w_{x}^{\cdot}\right)^{\cdot *} \varphi^{\cdot}+\left(g * \varphi^{\cdot}\right)^{\cdot *} \varphi^{\cdot}\right\} \\
& (x, s) d x d s \\
& =\int_{0}^{1}\left\{-Q\left(w_{x}^{\cdot}, \varphi^{\cdot *}\right)+\left(b * w_{x}^{\cdot}\right) \varphi^{\cdot}+\left(g * \varphi^{\cdot *}\right) \varphi^{\cdot}\right\}(x, t) d x \\
& -\int_{0}^{1} b(t) w_{x}^{\cdot}(x, t) \varphi^{\cdot}(x, 0) d x \\
& +\int_{0}^{t} \int_{0}^{1}\left\{b^{\prime}(s) w_{x}^{\cdot}(x, s) \varphi^{\cdot}(x, 0)+g^{\prime}(s) \varphi^{\cdot}(x, s) \varphi^{\cdot}(x, 0)\right\} d x d s,
\end{aligned}
$$

the final result is the energy equation of first order:

$$
\mathcal{U}\left(w^{\cdot}, \varphi^{\cdot}\right)+\mathcal{V}\left(w^{\cdot}, \varphi^{\cdot}\right)=\mathcal{F}\left(F^{\cdot}, R^{\cdot}, W^{\cdot}, \varphi^{\cdot}\right)+\mathcal{C}(w, \varphi)
$$

where

$$
\begin{aligned}
\mathcal{C}(w, \varphi)= & \frac{1}{2} \int_{0}^{1}\left(w^{\cdot 2}+G \varphi^{\cdot 2}\right)(x, 0) d x-\int_{0}^{1} b(t) w_{x}^{\cdot}(x, t) \varphi^{\cdot}(x, 0) d x \\
& +\int_{0}^{t} \int_{0}^{1}\left\{b^{\prime}(s) w_{x}^{\cdot}(x, s) \varphi^{\cdot}(x, 0)+g^{\prime}(s) \varphi^{\cdot}(x, s) \varphi^{\cdot}(x, 0)\right\} d x d s .
\end{aligned}
$$


To obtain our final energy equation we apply $\delta_{h}$ to (4.9), multiply the resulting equations by $\delta_{h} w^{\prime \prime}$ and $\delta_{h} \varphi^{\prime}$, proceed as in the derivation of (4.11), and then let $h \downarrow 0$. The outcome of this tedious but straightforward computation is the energy equation of second order,

$$
\begin{aligned}
& \mathcal{U}\left(w^{*}, \varphi^{*}\right)+\lim _{h \downarrow 0} \mathcal{V}\left(\delta_{h} w^{\prime}, \delta_{h} \varphi^{\cdot}\right) \\
& \quad=\mathcal{F}\left(F^{*}, R^{*}, w^{*}, \varphi^{\cdot *}\right)+\mathcal{C}\left(w^{*}, \varphi^{\cdot}\right)+\mathcal{D}(w, \varphi),
\end{aligned}
$$

where

$$
\begin{aligned}
& \mathcal{D}(w, \varphi)(t)=\int_{0}^{1}\left\{\left(\frac{1}{2} A+a\right) w_{x}^{. \cdot 2}+b w_{x}^{. \cdot} \varphi^{. \cdot}\right\}(x, 0) d x \\
& -\int_{0}^{1} a(t) w_{x}^{\ddot{*}}(x, t) w_{x}^{\ddot{*}}(x, 0) d x \\
& +\int_{0}^{t} \int_{0}^{1}\left\{a^{\prime}(s) w_{x}^{\cdot}(x, s) w_{x}^{\cdot}(x, 0)+b^{\prime}(s) w^{\cdots}(x, s) \varphi_{x}^{\cdot}(x, 0)\right. \\
& \left.+b^{\prime}(s) w_{x}^{\cdot}(x, 0) \varphi^{\cdot *}(x, s)+g^{\prime \prime}(s) \varphi^{\cdot}(x, s) \varphi^{\cdot}(x, 0)\right\} d x d s .
\end{aligned}
$$

$\left(\mathcal{V}\left(\delta_{h} w^{\prime}, \delta_{h} \varphi^{\cdot}\right)\right.$ has a limit as $h \downarrow 0$ because all of the other limits in the derivation of (4.13) exist; it then follows from (2.36) that $\mathcal{Q}\left(\delta_{h} w_{x}^{*}, \delta_{h} \varphi^{*}\right)$ has a limit as $h \downarrow 0$.)

REMARK 4A. The first- and second-order energy equations involve derivatives of $\varphi$ and $w$ at $t=0$; by (4.1), (4.4) $)_{2},(4.6)_{1}$, and (4.9):

$$
\begin{aligned}
& \varphi^{\cdot}(x, 0) \quad \text { depends linearly on } R(x, 0), \\
& w^{\prime \prime}(x, 0) \quad \text { depends linearly on } F(x, 0), \\
& \varphi^{*}(x, 0) \quad \text { depends linearly on } R(x, 0), R^{\cdot}(x, 0), R_{x x}(x, 0), F_{x}(x, 0), \\
& w^{\cdots}(x, 0) \quad \text { depends linearly on } R_{x}(x, 0), F^{\cdot}(x, 0) .
\end{aligned}
$$

4.3. The initial estimate. The three energy equations derived above yield a useful estimate. To derive this and remaining results we will frequently use the inequalities

$$
\begin{aligned}
& |X Y| \leq \frac{1}{2}\left(X^{2}+Y^{2}\right), \quad\left(\sum_{i=1}^{N} X_{i}\right)^{2} \leq N \sum_{i=1}^{N} X_{i}^{2}, \\
& |X Y| \leq \eta X^{2}+(4 \eta)^{-1} Y^{2}
\end{aligned}
$$

in conjunction with the following notation: 


$$
\begin{aligned}
\mathcal{E}(w, \varphi)(T)= & \max _{t \in[0, T]} \int_{0}^{1}\left\{w^{2}+\left|D^{1} w\right|^{2}+\left|D^{2} w\right|^{2}+\left|D^{3} w\right|^{2}\right. \\
& \left.+\varphi^{2}+\left|D^{1} \varphi\right|^{2}+\left|D^{2} \varphi\right|^{2}+\varphi_{x x x}^{2}+\varphi_{x x}^{\cdot 2}\right\}(x, t) d x \\
& +\int_{0}^{T} \int_{0}^{1}\left\{w^{2}+\left|D^{1} w\right|^{2}+\left|D^{2} w\right|^{2}+\left|D^{3} w\right|^{2}+\varphi^{2}\right. \\
& \left.+\left|D^{1} \varphi\right|^{2}+\left|D^{2} \varphi\right|^{2}+\varphi_{x x x}^{2}+\varphi_{x x}^{\cdot 2}+\varphi_{x}^{. \cdot 2}\right\}(x, t) d x d t,
\end{aligned}
$$

$$
\begin{aligned}
\mathcal{R}(F, R)(T)= & \max _{t \in[0, T]} \int_{0}^{1}\left\{F^{2}+\left|D^{1} F\right|^{2}+R^{2}+\left|D^{1} R\right|^{2}\right\}(x, t) d x \\
& +\int_{0}^{1} R_{x x}^{2}(x, 0) d x \\
& +\int_{0}^{T} \int_{0}^{1}\left\{F^{2}+\left|D^{1} F\right|^{2}+R^{2}+\left|D^{1} R\right|^{2}+F^{\cdots \cdot 2}+R^{\cdot 2}\right\} \\
& (x, t) d x d t .
\end{aligned}
$$

Let $\Gamma$ denote a generic strictly positive constant which depends at most on the coefficients and kernels of (4.1) (and not on the time interval $[0, T]$ under consideration). We then have the following estimates for the terms in (4.8) (where the estimate for $\mathcal{V}(w, \varphi)(t)$ follows from Lemma 3D):

$\mathcal{U}(w, \varphi)(t) \geq \Gamma^{-1}\left\{\int_{0}^{1}\left(w^{-2}+w_{x}^{2}\right)(x, t) d x+\int_{0}^{t} \int_{0}^{1} \varphi_{x}^{2}(x, s) d x d s\right\}$,

$$
\begin{aligned}
\mathcal{V}(w, \varphi)(t) \geq & \Gamma^{-1} \int_{0}^{1}\left[\varphi^{2}+Q\left(w_{x}^{\cdot}, \varphi^{\cdot}\right)\right](x, t) d x, \\
\mathcal{F}(F, R, w, \varphi)(t) \leq & {[\mathcal{R}(F, R)(t) \mathcal{E}(w, \varphi)(t)]^{\frac{1}{2}} } \\
& \leq[\mathcal{R}(F, R)(T) \mathcal{E}(w, \varphi)(T)]^{\frac{1}{2}} \quad \forall t \in[0, T] .
\end{aligned}
$$


We therefore conclude from (4.8) that

$$
\begin{aligned}
\int_{0}^{1}\left\{w^{\cdot 2}+w_{x}^{2}\right. & \left.+\varphi^{2}+Q\left(w_{x}^{\cdot}, \varphi^{\cdot}\right)\right\}(x, t) d x \\
& +\int_{0}^{t} \int_{0}^{1} \varphi_{x}^{2}(x, s) d x d s \leq \Gamma \mathcal{B}(T) \quad \forall t \in[0, T],
\end{aligned}
$$

where

$$
\mathcal{B}(T)=\mathcal{R}(F, R)(T)+[\mathcal{R}(F, R)(T) \mathcal{E}(w, \varphi)(T)]^{\frac{1}{2}} .
$$

In what follows we will generally omit the quantification " $\forall t \in[0, T]$ " in assertions concerning $t$ and $T$. Consider next the first-order energy equation (4.11). Estimates of the form (4.18)-(4.20) hold for the first three terms, which leaves only $\mathcal{C}(w, \varphi)(t)$ to estimate. This term is estimated using Remark 4A to express the terms involving $w$ and $\varphi$ and their derivatives at $t=0$ in terms of $F$ and $R$ and their derivatives at $t=0$. A typical term of $\mathcal{C}(w, \varphi)(t)$ is estimated as follows:

$$
\begin{aligned}
& \left|\int_{0}^{t} \int_{0}^{1} b^{\prime}(s) w_{x}^{\cdot}(x, s) \varphi \cdot(x, 0) d x d s\right| \\
& \leq\left\{\int_{0}^{t} \int_{0}^{1} w_{x}^{\cdot 2}(x, s) d x d s\right\}^{\frac{1}{2}}\left\{\int_{0}^{t} \int_{0}^{1} b^{\prime}(s)^{2} R^{2}(x, 0) d x d s\right\}^{\frac{1}{2}} \\
& \leq[\mathcal{R}(F, R)(T) \mathcal{E}(w, \varphi)(T)]^{\frac{1}{2}} .
\end{aligned}
$$

(Note that $b, b^{\prime}, b^{\prime \prime} \in L^{p}[0, \infty)$ for all $p \in[1, \infty]$, since $b \in W^{3,1}[0, \infty)$.) The final result is

$$
\begin{gathered}
\int_{0}^{1}\left\{w^{\cdot \cdot 2}+w_{x}^{\cdot 2}+\varphi^{\cdot 2}+Q\left(w_{x}^{. \cdot}, \varphi^{\cdot *}\right)\right\}(x, t) d x+\int_{0}^{t} \int_{0}^{1} \varphi_{x}^{\cdot 2}(x, s) d x d s \\
\leq \Gamma \mathcal{B}(T) .
\end{gathered}
$$

Similarly, the second-order energy equation (4.13) yields the estimate

$$
\begin{aligned}
\int_{0}^{1}\left(w^{\cdots 2}+\right. & \left.w_{x}^{\cdot 2}+\varphi^{\cdot .2}\right)(x, t) d x+\int_{0}^{t} \int_{0}^{1} \varphi_{x}^{.2}(x, s) d x d s \\
+ & \lim _{h \downarrow 0} \mathcal{Q}\left(\delta_{h} w_{x}^{\cdot .}, \delta_{h} \varphi^{\cdot *}\right) \leq \Gamma \mathcal{B}(T) .
\end{aligned}
$$


Combining the estimates (4.21)-(4.24), we find, with the aid of Lemma $3 \mathrm{~A}$ and the initial conditions (4.4), that

$$
\begin{aligned}
& \int_{0}^{1}\left\{\left|D^{1} w\right|^{2}+w_{x}^{.2}+w^{. \cdot 2}+w_{x}^{.2}+w^{\cdots 2}+\varphi^{2}+\varphi^{\cdot 2}+\varphi^{. \cdot 2}\right\}(x, t) d x \\
& +\int_{0}^{t} \int_{0}^{1}\left\{w_{x}^{.2}+w_{x}^{. \cdot 2}+\left|D^{1} \varphi\right|^{2}+\varphi_{x}^{.2}+\varphi^{. \cdot 2}+\varphi_{x}^{.2}\right\}(x, s) d x d s \leq \Gamma \mathcal{B}(T),
\end{aligned}
$$

and we may use Poincare's inequality and the inequality

$$
\int_{0}^{1} \psi^{2}(x, t) d x \leq \int_{0}^{1} \psi^{2}(x, 0) d x+\int_{0}^{t} \int_{0}^{1}\left(\psi^{2}+\psi^{\cdot 2}\right)(x, s) d x d s
$$

to conclude that

$$
\begin{gathered}
\int_{0}^{1}\left\{w^{2}+\left|D^{1} w\right|^{2}+w_{x}^{.2}+w^{. \cdot 2}+w_{x}^{. \cdot 2}+w^{\cdots 2}+\varphi^{2}+\left|D^{1} \varphi\right|^{2}+\varphi_{x}^{\cdot 2}+\varphi^{. \cdot 2}\right\} \\
(x, t) d x \\
+\int_{0}^{t} \int_{0}^{1}\left\{w^{\cdot 2}+w_{x}^{.2}+w^{. \cdot 2}+w_{x}^{. \cdot 2}+\varphi^{2}+\left|D^{1} \varphi\right|^{2}+\varphi_{x}^{\cdot 2}+\varphi^{. \cdot 2}+\varphi_{x}^{. \cdot 2}\right\} \\
\leq \Gamma \mathcal{B}(T) .
\end{gathered}
$$

Our final step will be to obtain estimates for the remaining terms in $\mathcal{E}(w, \varphi)$.

4.4 The final estimate. In this section we derive the estimate

$$
\mathcal{E}(w, \varphi)(T) \leq \Gamma \mathcal{R}(F, R)(T) .
$$

We estimate those derivatives in $\mathcal{E}(w, \varphi)$ that are not present in $(4.27)$ using the equations (4.1) in conjunction with Lemma $3 \mathrm{E}$. Here it is convenient to use the notation

$$
\mathcal{M}(\mathrm{f})(t)=\int_{0}^{1} \mathrm{f}^{2}(x, t) d x+\int_{0}^{t} \int_{0}^{1} \mathrm{f}^{2}(x, s) d x d s
$$

By $(4.1)_{2}$,

$$
\varphi_{x x}=K^{-1}\left\{(G-g(0)) \varphi^{\cdot}-(B+b(0)) w_{x}^{\cdot}-b^{\prime} * w_{x}^{\cdot}-g^{\prime} * \varphi^{\cdot}-R\right\}
$$


and, since $b^{\prime}, g^{\prime} \in L^{1}(0, \infty)$, we may use (4.27) to conclude that

$$
\mathcal{M}\left(\varphi_{x x}\right)(t) \leq \Gamma \mathcal{B}(T)
$$

Similarly, by solving $(4.9)_{2}$ for $\varphi_{x x}^{\cdot}$, we find that

$$
\mathcal{M}\left(\varphi_{x x}^{\cdot}\right)(t) \leq \Gamma \mathcal{B}(T) .
$$

Next, by $(4.1)_{1}$,

$$
(A+a(0)) w_{x x}+a^{\prime} * w_{x x}=w^{*}-(B+b(0)) \varphi_{x}-b^{\prime} * \varphi_{x}-F
$$

and we may use Lemma $3 \mathrm{E}$ and (4.27) to show that

$$
\mathcal{M}\left(w_{x x}\right)(t) \leq \Gamma \mathcal{B}(T) .
$$

Similarly, differentiating $(4.1)_{1}$ with respect to $x$, we conclude, with the aid of Lemma 3E, (4.27), and (4.31), that

$$
\mathcal{M}\left(w_{x x x}\right)(t) \leq \Gamma \mathcal{B}(T),
$$

and using Lemma $3 \mathrm{E}$ to solve $(4.9)_{1}$ for $w_{x x}^{\circ}$, we find, using (4.27), that

$$
\int_{0}^{1} w_{x x}^{\cdot 2}(x, t) d x \leq \Gamma \mathcal{B}(T) .
$$

Next, we record the interpolation inequality

$$
\begin{aligned}
\int_{0}^{t} \int_{0}^{1} w_{x x}^{\cdot 2}(x, s) d x d s & \\
\leq \Gamma\left\{\int _ { 0 } ^ { t } \int _ { 0 } ^ { 1 } \left\{w_{x x x}^{2}\right.\right. & \left.+w_{x}^{\cdot 2}+w_{x x}^{2}\right\}(x, s) d x d s \\
& \left.+\int_{0}^{1}\left\{w_{x}^{\cdot 2}+w_{x x}^{2}+w_{x x x}^{2}\right\}(x, t) d x\right\}
\end{aligned}
$$

(To establish (4.7): extend $w$ to all of space by $H^{3}$-reflection (cf., e.g., $[\mathbf{9}, \S 1.2 .2])$ and multiplication by a smooth spatial cutoff function, 
integrate by parts, restrict the result to $[0,1]$, and use Poincare's inequality.) By virtue of (4.27), (4.34), and (4.35), (4.37) yields

$$
\int_{0}^{t} \int_{0}^{1} w_{x x}^{\cdot 2}(x, s) d x d s \leq \Gamma \mathcal{B}(T)
$$

Finally, we use $(4.9)_{1},(4.27)$, and (4.38) to conclude that

$$
\int_{0}^{t} \int_{0}^{1} w^{\cdots 2}(x, s) d x d s \leq \Gamma \mathcal{B}(T)
$$

and we differentiate $(4.1)_{2}$ with respect to $x$, solve the resulting equation for $\varphi_{x x x}$, and use (4.27), (4.36), and (4.38) to obtain

$$
\mathcal{M}\left(\varphi_{x x x}\right)(t) \leq \Gamma \mathcal{B}(T) .
$$

Adding (4.27), (4.31), (4.32), (4.34)-(4.36), and (4.38)-(4.40), and using Poincare's inequality, we arrive at the inequality

$$
\mathcal{E}(w, \varphi)(T) \leq \Gamma \mathcal{B}(T) .
$$

The desired estimate (4.28) follows from (4.22), (4.41), and

$$
[\mathcal{R}(F, R)(T) \mathcal{E}(w, \varphi)(T)]^{\frac{1}{2}} \leq \eta \mathcal{E}(w, \varphi)(T)+(4 \eta)^{-1} \mathcal{R}(F, R)(T),
$$

with $\eta$ sufficiently small.

5. The linear system with nonconstant coefficients. In this section we will establish a priori estimates for the linear equations (2.32) (on a fixed time interval $[0, T]$ ) with coefficients

$$
A(x, t), \quad B(x, t), \quad G(x, t), \quad K(x, t), \quad S(x, t)
$$

and supply terms

$$
F(x, t), \quad R(x, t)
$$

allowed to be functions of $(x, t) \in[0,1] \times[0, T]$ :

$$
\begin{aligned}
w^{*} & =A w_{x x}+B \varphi_{x}+\left(a * w_{x x}\right)^{\cdot}+\left(b * \varphi_{x}\right)^{\cdot}+F \\
G \varphi^{\cdot} & =\left(K \varphi_{x}\right)_{x}+B w_{x}^{\cdot}+\left(b * w_{x}^{\cdot}\right)^{\cdot}+\left(g * \varphi^{\cdot}\right)^{\cdot}+S w_{x}^{\cdot}+R .
\end{aligned}
$$


We assume that $F$ and $R$ satisfy (4.2). In addition, we require that the coefficients satisfy the boundedness conditions

$$
\begin{aligned}
0<\alpha \leq A(x, t), \quad G(x, t)-g(0), \quad K(x, t) \leq \beta, \quad|B(x, t)| \leq \beta, \\
\\
\forall(x, t) \in[0,1] \times[0, T],
\end{aligned}
$$

as well as the smoothness requirements

$$
A, D^{1} A, D^{2} A, B, D^{1} B, D^{2} B, G, D^{1} G, D^{2} G,
$$

$$
K, D^{1} K, D^{2} K, S, D^{1} S, D^{2} S \in C\left([0, T] ; L^{2}(0,1)\right),
$$

and we measure the influence of the coefficients using the norm

$$
\begin{aligned}
& \delta(T)=\max _{[0,1] \times[0, T]}\left\{\left|A-A_{0}\right|+\left|D^{1} A\right|+\left|D^{1} B\right|+\left|D^{1} G\right|\right. \\
& \left.+\left|D^{1} K\right|+|S|+\left|D^{1} S\right|\right\} \\
& +\int_{0}^{T} \int_{0}^{1}\left\{A^{\cdot \cdot 2}+B^{\cdot \cdot 2}+G^{\cdot 2}+K^{\cdot \cdot 2}+K_{x}^{\cdot 2}+S^{\cdots 2}\right\}^{\frac{1}{2}}(x, t) d x d t
\end{aligned}
$$

with

$$
A_{0}=\int_{0}^{1} A(x, 0) d x .
$$

Note that if $A, B, G$, and $K$ are constant and $S=0$, then $\delta(T)=0$.

LEMma 5A. Let constants $\alpha, \beta>0$ and kernels $a, b, g \in C^{3}[0, \infty) \cap$ $W^{3,1}[0, \infty)$ consistent with (A5) be given. Then there is a constant $\Gamma$ that is independent of $T$, the coefficients, and the supply terms, such that if $A, B, G, K$ and $S$ obey (5.2) and (5.3), if $F$ and $R$ obey (4.2), and if $(w, \varphi)$ satisfies (4.4), (4.5), and (5.1), then the estimate

$$
\mathcal{E}(w, \varphi)(T) \leq \Gamma \mathcal{R}(F, R)(T)+\Gamma\left[\delta(T)+\delta(T)^{2}\right] \mathcal{E}(w, \varphi)(T)
$$

holds with $\mathcal{E}, \mathcal{R}$, and $\delta$ given by (4.16), (4.17), and (5.4).

The proof of this lemma is not much different from that of (4.28). Since the coefficients are not constant, the relations (4.8), (4.11), and 
(4.13) will contain additional terms. Defining $\mathcal{N}_{0}(w, \varphi)$ through $(2.36)_{4}$ and writing

$$
\begin{aligned}
& \mathcal{N}_{1}(w, \varphi)(t)=\int_{0}^{t} \int_{0}^{1}\left\{A^{\cdot} w_{x x} w^{\cdot *}+B^{\cdot}\left(\varphi_{x} w^{\cdot *}+w_{x}^{\cdot} \varphi^{\cdot}\right)+K^{\cdot} \varphi_{x x} \varphi^{\cdot}\right. \\
& \left.+K_{x}^{*} \varphi_{x} \varphi^{\cdot}-G^{\cdot} \varphi^{\cdot 2}+S w_{x}^{\cdot} \varphi^{\cdot}\right\}(x, s) d x d s \\
& \mathcal{N}_{2}(w, \varphi)(t)=\int_{0}^{t} \int_{0}^{1}\left\{A^{*} w_{x x} w^{\cdots}+B^{*}\left(\varphi_{x} w^{\cdots}+w_{x}^{\cdot} \varphi^{*}\right)\right.
\end{aligned}
$$

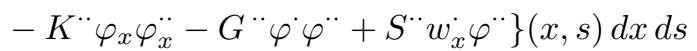

(4.8), (4.11), and (4.13) should have

$$
\begin{gathered}
\mathcal{N}_{0}(w, \varphi), \quad \mathcal{N}_{0}\left(w^{\prime}, \varphi^{\cdot}\right)+\mathcal{N}_{1}(w, \varphi), \\
\mathcal{N}_{0}\left(w^{*}, \varphi^{\cdot *}\right)+2 \mathcal{N}_{1}\left(w^{\prime}, \varphi^{\cdot}\right)+\mathcal{N}_{2}(w, \varphi),
\end{gathered}
$$

respectively, added to their right sides. Each of these is bounded by

$$
\Gamma \delta(T) \mathcal{E}(w, \varphi)(T),
$$

and thus (4.27) should have (5.7) added to its right side. Continuing to follow the analysis of Section 4, we see that (4.31) also should have (5.7) on its right side, while (4.32) should have

$$
\Gamma\left[\delta(T)+\delta(T)^{2}\right] \mathcal{E}(w, \varphi)(T)
$$

on the right. Further, writing the first term in (4.33) in the form

$$
\left(A_{0}+a(0)\right) w_{x x}+\left(A-A_{0}\right) w_{x x},
$$

leads to (4.34) supplemented by (5.8), and (5.8) should also be added to the right sides of (4.35), (4.36), (4.38), (4.39), and (4.40), and finally to the right side of (4.28), so that (5.5) is satisfied.

To give an indication of the additional steps involved in the derivation of (5.5), we now give detailed estimates for two of the terms in (5.6); the remaining terms are treated analogously. In these calculations the symbol "max" designates "maximum over $[0,1] \times[0, T]$ " and $t \in[0, T]$. The first term we consider is handled in a simple fashion

$$
\begin{aligned}
\left|\int_{0}^{t} \int_{0}^{1}\left(A^{\cdot} w_{x x}^{\cdot} w^{\cdots}\right)(x, s) d x d s\right| & \leq\left(\max \left|A^{\cdot}\right|\right) \int_{0}^{t} \int_{0}^{1}\left|w_{x x}^{\cdot} w^{\cdots}\right|(x, s) d x d s \\
& \leq \frac{1}{2} \delta(T) \int_{0}^{t} \int_{0}^{1}\left(w_{x x}^{\cdot 2}+w^{\cdots 2}\right)(x, s) d x d s \\
& \leq \frac{1}{2} \delta(T) \mathcal{E}(w, \varphi)(T) .
\end{aligned}
$$


Terms involving a twice-differentiated coefficient are treated in a slightly different manner; e.g.,

$$
\begin{aligned}
& \left|\int_{0}^{t} \int_{0}^{1}\left(A^{*} w_{x x} w^{\cdots}\right)(x, s) d x d s\right| \leq\left(\max \left|w_{x x}\right|\right) \int_{0}^{t} \int_{0}^{1}\left|A^{* *} w^{\cdots}\right|(x, s) d x d s \\
& \leq\left(\max \left|w_{x x}\right|\right)\left(\int_{0}^{t} \int_{0}^{1} A^{\cdots 2}(x, s) d x d s\right)^{\frac{1}{2}}\left(\int_{0}^{t} \int_{0}^{1} w^{\cdots 2}(x, s) d x d s\right)^{\frac{1}{2}} .
\end{aligned}
$$

The Sobolev embedding theorem implies that, for $\xi \in[0,1]$,

$$
w_{x x}^{2}(\xi, t) \leq 2 \int_{0}^{1}\left(w_{x x}^{2}+w_{x x x}^{2}\right)(x, s) d x d s \leq 2 \mathcal{E}(w, \varphi)(T),
$$

so that (5.9) yields

$$
\left|\int_{0}^{t} \int_{0}^{1}\left(A^{*} w_{x x} w^{\cdots}\right)(x, s) d x d s\right| \leq \sqrt{2} \delta(T) \mathcal{E}(w, \varphi)(T) .
$$

6. Global existence for the nonlinear problem. Consider the history-value problem defined by (2.1)-(2.5) and assume that (A1)-(A5) hold. The existence of a local solution can be established by a standard contraction-mapping argument and we omit the details; the relevant result is recorded in the proposition below. Since we have made assumptions concerning the signs of the coefficients only at the equilibrium state $\left(0, \theta_{0}\right)$, we must ensure that $\left(u_{x}, \theta\right)$ remains in a suitable neighborhood of $\left(0, \theta_{0}\right)$. We choose $d>0$ and $\alpha>0$ such that $d<1, d<\theta_{0}$, and

$$
\begin{gathered}
A\left(\varepsilon^{t}, \theta^{t}\right), G\left(\varepsilon^{t}, \theta^{t}\right)-g(0), K(\varepsilon(t), \theta(t)) \geq \alpha, \\
\forall(\varepsilon, \theta) \in C\left(\mathbf{R} ; U_{d}\right), t \in \mathbf{R},
\end{gathered}
$$

where

$$
U_{d}=(-d, d) \times\left(\theta_{0}-d, \theta_{0}+d\right) .
$$

We seek a solution to (2.30) subject to the conditions:

$$
\begin{aligned}
u(0, t) & =u(1, t)=0, \quad \theta(0, t)=\theta(1, t)=\theta_{0}, \quad t \geq 0, \\
u(x, 0) & =u(x, 0)=0, \quad \theta(x, 0)=\theta_{0}, \quad x \in[0,1] .
\end{aligned}
$$


Proposition. For every $f, r$ consistent with (A6), the problem (2.30), (6.1) has a unique solution $(u, \theta)$ on a maximal time interval $\left[0, T_{0}\right), 0<$ $T_{0}<\infty$, with

$$
\begin{gathered}
u, D^{1} u, D^{2} u, \theta-\theta_{0}, D^{1} \theta, D^{2} \theta, \theta_{x x x}, \theta_{x x} \in C\left(\left[0, T_{0}\right) ; L^{2}(0,1)\right), \\
\theta_{x}^{*} \in L_{\text {loc }}^{2}\left(\left[0, T_{0}\right) ; L^{2}(0,1)\right), \\
\left(u_{x}(x, t), \theta(x, t)\right) \in U_{d}, \quad \forall(x, t) \in[0,1] \times\left[0, T_{0}\right) .
\end{gathered}
$$

Moreover (for $\mathcal{E}$ as defined in (4.16)), if

$$
\sup _{t \in\left[0, T_{0}\right)} \mathcal{E}\left(u, \theta-\theta_{0}\right)(t)<\infty, \sup _{[0,1] \times\left[0, T_{0}\right)}\left|u_{x}\right|<\delta, \sup _{[0,1] \times\left[0, T_{0}\right)}\left|\theta-\theta_{0}\right|<\delta,
$$

then $T_{0}=\infty$.

The proof of this proposition is very similar to the isothermal case (cf., e.g., $[\mathbf{3}, \mathbf{1 3}])$. Local existence in three-dimensional nonlinear viscoelasticity will be discussed in a forthcoming paper of Messaoudi [10].

Let $f$ and $r$ satisfying (A6) be given, and consider the local solution $(u, \theta)$ of $(2.30),(6.1)$. Our objective is to show that if (2.27) holds with $\mu$ sufficiently small, then

$$
\sup _{t \in\left[0, T_{0}\right)} \mathcal{E}\left(u, \theta-\theta_{0}\right)(t)<d^{2} .
$$

Since, by the Sobolev embedding theorem,

$$
\left\{u^{2}+\left(\theta-\theta_{0}\right)^{2}\right\}(x, t) \leq \mathcal{E}\left(u, \theta-\theta_{0}\right)(t), \quad \forall(x, t) \in[0,1] \times\left[0, T_{0}\right),
$$

the inequality (6.2) implies $T_{0}=\infty$.

We define $F$ and $R$ through (2.33) and identify $A(x, t)$ in (5.1) with $A\left(u_{x}^{t}, \theta^{t}\right)$, etc. Then, for every $T \in\left(0, T_{0}\right)$, we may apply Lemma $5 \mathrm{~A}$ with $w=u$ and $\varphi=\theta-\theta_{0}$.

Let us agree to write $\mathcal{E}(T)$ in place of $\mathcal{E}\left(u, \theta-\theta_{0}\right)(T)$. It follows from the Sobolev embedding theorem that

$$
\begin{gathered}
\left\{u^{2}+\left|D^{1} u\right|^{2}+\left|D^{2} u\right|^{2}+\left(\theta-\theta_{0}\right)^{2}+\left|D^{1} \theta\right|^{2}+\theta_{x x}^{2}+\theta_{x}^{\cdot 2}\right\}(x, t) \leq \Gamma \mathcal{E}(t), \\
\forall(x, t) \in[0,1] \times\left[0, T_{0}\right) .
\end{gathered}
$$


Since the closure of $U_{d}$ is a compact subset of $U$, we may use (A1), (6.3), and Lemma 5A to arrive at an inequality of the form

$$
\mathcal{E}(T) \leq \Gamma_{1} \mathcal{J}(f, r)+H(\mathcal{E}(T)) \mathcal{E}(T), \quad \forall T \in\left[0, T_{0}\right),
$$

where $\mathcal{J}$ is defined by $(2.26), H:[0, \infty) \rightarrow[0, \infty)$ is a continuous function with $H(0)=0$, and $\Gamma_{1}$ is a positive constant that is independent of $T_{0}$ and the data. (Note that, by the Sobolev embedding theorem, $\mathcal{R}(f, r)(T) \leq \Gamma \mathcal{J}(f, r)$ for all $T \geq 0$.) The derivation of (6.4) is straightforward, but tedious because of the number of terms involved; we refer to $[\mathbf{3}]$ and $[\mathbf{1 3}]$ for the details of similar calculations.

We choose $\mathcal{E}_{0}, \mu>0$ such that

$$
H(z) \leq \frac{1}{2}, \quad \forall z \in\left[0, \mathcal{E}_{0}\right], \quad \mathcal{E}_{0} \leq \frac{1}{2} d^{2}, \quad \Gamma \mu \leq \frac{1}{4} \mathcal{E}_{0},
$$

and such that

$$
\mathcal{J}(f, r) \leq \mu \Rightarrow \mathcal{E}(0) \leq \frac{1}{2} \mathcal{E}_{0} .
$$

Suppose that (2.27) holds with $\mu$ as above. It follows from (6.4) and (6.5) that, for each $T \in\left[0, T_{0}\right)$ with $\mathcal{E}(T) \leq \mathcal{E}_{0}$, we actually have $\mathcal{E}(T) \leq \mathcal{E}_{0} / 2$. Consequently, by (6.6) and the continuity of $\mathcal{E}$ we have $\mathcal{E}(T) \leq \mathcal{E}_{0} / 2$ for all $T \in\left[0, T_{0}\right)$. Since $\mathcal{E}_{0} \leq d^{2} / 2$, it follows that $(6.11)$ holds and hence that $T_{0}=\infty$. The boundedness of $\mathcal{E}$ on $[0, \infty)$ yields the additional bounds and the decay of the solution as asserted in the theorem.

Acknowledgments. We are grateful to D. Brandon, S.O. Londen, V.J. Mizel, and A. Struthers for valuable discussions and helpful suggestions. This work was supported by the Air Force Office of Scientific Research, the Army Research Office, and the National Science Foundation.

\section{REFERENCES}

1. D. Brandon, Global existence and asymptotic stability for a nonlinear integrodifferential equation modelling heat flow, SIAM J. Math. Anal., to appear.

2. B.D. Coleman, Thermodynamics of materials with memory, Arch. Rational Mech. Anal. 17 (1964), 1-46. 
3. C.M. Dafermos and J.A. Nohel, A nonlinear hyperbolic Volterra equation in viscoelasticity, Amer. J. Math. Suppl. (1981), 87-116.

4. G. Gripenberg, S. Londen, and O. Staffans, Volterra integral and functional equation, Cambridge Univ. Press, to appear.

5. M.E. Gurtin and I. Herrera, On dissipation inequalities and linear viscoelasticity, Quart. Appl. Math. 23 (1965) 235-245.

6. M.E. Gurtin and W.J. Hrusa, On the thermodynamics of viscoelastic materials of single-integral type, Quart. Appl. Math., to appear.

7. W.J. Hrusa and J.A. Nohel, The Cauchy problem in one-dimensional nonlinear viscoelasticity, J. Differential Equations 59 (1985), 388-412.

8. W.J. Hrusa and M.A. Tarabek, On smooth solutions of the Cauchy problem in one-dimensional nonlinear thermoelasticity, Quart. Appl. Math. 47 (1989), 631-644.

9. J.L. Lions and E. Magenes, Non-Homogeneous boundary value problems and applications, Springer, New York, 1972.

10. S. Messaoudi, A local existence theorem in three-dimensional nonlinear thermoviscoelasticity, forthcoming.

11. C.B. Navarro, Asymptotic stability in linear thermoviscoelasticity, J. Math. Anal. Appl. 65 (1978), 399-431.

12. - Existence and uniqueness in the Cauchy problem for a linear thermoelastic material with memory, in Heriot-Watt Sympos. on Nonlinear Analysis and Mechanics (R.J. Knops, ed.), Pitman Res. Notes Math. Ser., vol. 27, Longman Sci. Tech., Harlow, 1978, pp. 191-204 (Appendix to contribution of J.E. Marsden and T.J.R. Hughes).

13. M. Renardy, W.J. Hrusa, and J.A. Nohel, Mathematical problems in viscoelasticity, Longman Sci. Tech., Harlow, and John Wiley, New York, 1987.

14. M. Slemrod, Global existence, uniqueness, and asymptotic stability of classical smooth solutions in one-dimensional nonlinear thermoelasticity, Arch. Rational Mech. Anal. 76 (1981), 97-133.

15. O.J. Staffans, On a nonlinear hyperbolic Volterra equation, SIAM J. Math. Anal. 11 (1980), 793-812.

16. S. Zheng and W. Shen, Global solutions to the Cauchy problem of a class of hyperbolic-parabolic coupled systems, Internat. Workshop on Applied Differential Equations (S.T. Xiao and F.Q. Pu, eds.), World Scientific Publ., Singapore, 1986, pp. $335-338$.

Department of Mathematics, Carnegie Mellon University, Pittsburgh, PA 15213 